\title{
Nutmeg Oil
}

National Cancer Institute

\section{Source}

National Cancer Institute. Nutmeg Oil. NCI Thesaurus. Code C107336.

The essential oil obtained from ground nutmeg. Nutmeg oil is typically used as a food flavoring but also has analgesic properties. 